\title{
La importància de la informació audiovisual en l'adquisició del llenguatge
}

\section{Joan Birulés i Ferran Pons}

Departament de Cognició, Desenvolupament i Psicologia de l'Educació, Universitat de Barcelona, Institut de Neurociències (grup APAL)

joanbirules@ub.edu

ferran.pons@ub.edu

Recepció: 02/04/2019, acceptació: 13/05/2019

Resum: Tot i que els adults generalment mirem als ulls quan ens parlen, quan ens costa d'entendre allò que diuen movem l'atenció visual a la zona de la boca per ajudar-nos a comprendre millor el missatge. D'aquesta manera, integrem millor les pistes visuals $(i$. $e$. moviments articulatoris que encaixen temporalment i acústicament) amb els sons que escoltem, i formem un percepte audiovisual que ens és més senzill d'entendre que un percepte auditiu sol. Així mateix, els infants també centren la mirada en la boca d'un parlant a partir de la segona meitat del primer any de vida, la qual cosa s'ha interpretat com un reflex de l'ús creixent de les pistes audiovisuals, que els ajuda en el procés d'adquisició del llenguatge. En aquest article revisarem els estudis experimentals més destacats sobre el paper de la informació audiovisual en el desenvolupament i l'adquisició del llenguatge, així com els factors que poden modular el processament de les pistes audiovisuals.

Paraules clau: percepció del llenguatge, percepció audiovisual, infància, atenció

\section{La importancia de la información audiovisual en la adquisición del lenguaje}

Resumen: Aunque los adultos generalmente miramos a los ojos cuando nos hablan, cuando nos cuesta de entender lo que dicen movemos la atención visual a la zona de la boca y eso nos ayuda a comprender mejor el mensaje. De esta manera integramos mejor las pistas visuales (i.e. movimientos articulatorios que encajan temporal y acústicamente) con los sonidos que escuchamos y formamos un percepto audiovisual que nos es más sencillo de entender que el auditivo solo. Paralelamente, los bebés también centran la mirada en la boca de un hablante a partir de la segunda mitad del primer año de vida, lo que se ha interpretado como un reflejo del uso creciente de las pistas audiovisuales, que les ayuda en el proceso de adquisición del lenguaje. En este artículo revisaremos los estudios experimentales más destacados sobre el papel de la información audiovisual en el desarrollo y la adquisición del lenguaje, así como los factores que pueden modular el procesamiento de las pistas audiovisuales.

Palabras clave: percepción del lenguaje, percepción audiovisual, infancia, atención 


\section{The relevance of audiovisual information in language acquisition}

Abstract: Although adults usually look at the eyes of talking faces, when the auditory information becomes compromised, they switch towards the mouth area to help them process the speech signal. In this way, the visual speech cues (i.e. articulatory movements that fit temporally and acoustically with the sounds produced) can be integrated with the speech sounds and form an audiovisual perception that is easier to understand than the auditory one alone. Likewise, infants also focus their attention on the talker's mouth from the second half of their first year of life. This has been interpreted to reflect the growing use of audiovisual speech cues, which helps them in the language acquisition process. In this article we will review the most important experimental studies on the role of audiovisual information in the development and acquisition of language, as well as the factors that can modulate the processing of audiovisual cues.

Keywords: Language perception, audiovisual perception, infancy, attention

\section{INTRODUCCIÓ}

La major part de les interaccions socials són cara a cara, és a dir, no només escoltem el missatge sinó que també veiem la cara de l'interlocutor. Veure la cara ens dona molta informació: d'una banda, ens ajuda a identificar el parlant i, de l'altra, gràcies als moviments facials podem inferir el seu estat mental, l'actitud i les intencions potencials (Birmingham i Kingstone 2009). Finalment, el més rellevant pel que fa al processament de la parla és que ens permet observar els moviments articulatoris de la boca que s'ajusten als sons produïts (Chandrasekaran, Trubanova, Stillittano, Caplier i Ghazanfar 2009; Yehia, Rubin i Vatikiotis-Bateson 1998). Diversos estudis demostren que processar conjuntament aquestes claus visuals i la informació auditiva forma un percepte nou, un senyal lingüístic que «sobresurt» més i que ens ajuda a percebre millor la parla (Meredith i Stein 1986; Risberg i Lubker 1978). Aquesta afirmació es basa en els resultats d'estudis clàssics en què es va demostrar que, efectivament, comprenem millor la parla presentada de manera audiovisual -comparat amb la presentació només auditiva- quan la informació auditiva és degradada, per exemple afegint-hi soroll (Cotton 1935, Sanders i Goodrich 1971, Sumby i Pollack 1954).

Tots aquests estudis clàssics indiquen que la informació audiovisual és molt important en el procés de la percepció i processament de la parla. En conseqüència, una de les qüestions suscitades en el camp de la psicologia del desenvolupament ha estat: és possible que la informació audiovisual tingui també un paper rellevant en els processos d'adquisició del llenguatge? Efectivament, estudis amb infants demostren que les pistes audiovisuals milloren la percepció, l'aprenentatge i l'adquisició del llenguatge (Bahrick i Lickliter 2012; Bremner, Lewkowicz i Spence 2012). En aquest article revisarem els estudis experimentals més destacats sobre el paper de la informació audiovisual en l'adquisició del llenguatge durant el desenvolupament.

\section{LA INTEGRACIÓ I LA CORRESPONDÈNCIA AUDIOVISUAL DE LA}

\section{PARLA DURANT EL PRIMER ANY DE VIDA}

És important considerar que el nadó no està únicament exposat a estímuls lingüístics auditius sinó que també en percep les correspondències visuals. Darrerament, aquest tipus d'informació visual que envolta el procés d'adquisició de la parla en nadons ha rebut especial atenció. Un dels estudis més rellevants sobre la importància d'aspectes no acústics en la percepció de la parla en nadons és el de Weikum i col-laboradors (2007), en 
el qual es va remarcar la importància d'estudiar com la informació visual influeix en l'adquisició i el desenvolupament de la parla, més enllà de l'aspecte auditiu. En aquest treball pioner, els autors van estudiar la capacitat dels infants de 4 a 8 mesos de diferenciar dues llengües mitjançant únicament la informació visual (i. e. gestos articulatoris de la cara). Els resultats d'aquest estudi van mostrar que els nadons més petits (4-6 mesos) són capaços de diferenciar la seva llengua materna d'una altra llengua usant únicament informació visual, però que aquesta habilitat es perd amb el pas del temps (als 8 mesos d'edat), llevat que l'infant estigui exposat a aquestes dues llengües (infants d'entorn bilingüe). D'aquesta manera es va demostrar la capacitat dels infants en percebre i utilitzar les pistes visuals de la parla durant el període d'adquisició del llenguatge.

És sorprenent que, tot i l'acord general en la rellevància d'estudiar l'adquisició del llenguatge des d'una perspectiva audiovisual, els mecanismes d'integració audiovisual de la parla en les primeres etapes del desenvolupament no estan encara del tot ben definits. Tot i això, els pocs estudis que han abordat la temàtica hi han fet aportacions molt importants. Per exemple, s'ha observat que els nadons de 2-4 mesos d'edat són capaços d'integrar presentacions auditives d'una vocal o d'una síl-laba amb els seus corresponents moviments labials o articulatoris (Kuhl i Meltzoff 1982, Patterson i Werker 2003). També, que a aquesta edat els nadons prefereixen atendre (mirar) la cara de la persona que està parlant quan el so i el moviment dels llavis són temporalment sincrònics (Dodd 1979). Més endavant, als 5-7 mesos d'edat, els infants ja són capaços d'integrar el contingut afectiu de les cares i les veus (Walker-Andrews 1997) i, als 7-9 mesos, de detectar el gènere de les cares i la veu corresponent (Poulin-Dubois, Serbin, Kenyon i Derbyshire 1994). D’altra banda, quan la informació auditiva i visual de la parla es troben en conflicte, fenomen conegut com efecte McGurk ${ }^{1}$, nadons de 4-5 mesos d'edat perceben la síl-laba en funció d'allò que se'ls presenta visualment (Rosenblum, Schmuckler i Johnson 1997). Finalment, cal destacar un estudi que va demostrar que el fenomen de la reorganització fonètica perceptiva durant el primer any de vida no succeeix únicament en el pla acústic o visual sinó que també s'observa en el pla intersensorial, és a dir, audiovisual. En aquest estudi es va observar que els nadons d'entorn lingüístic anglès integraven el so amb el seu corresponent moviment o gest articulatori d'un contrast fonètic de la seva llengua materna (anglès) tant als 6 com als 11 mesos, mentre que els nadons d'entorn lingüístic espanyol eren únicament capaços de percebre la correspondència auditiva i visual d'aquest contrast fonètic d'una llengua no materna (anglès) als 6 mesos, però no als 11 mesos, un cop ha finalitzat el procés de reorganització perceptiva dels sons de la llengua (Pons, Lewkowicz, Soto-Faraco i Sebastián-Gallés 2009). Un altre estudi que també remarca que l'experiència modula la correspondència audiovisual és el de Vouloumanos i col-laboradors (2009), en què demostren que els nadons de 5 mesos poden percebre la correspondència entre vocalitzacions i cares estàtiques de persones i de micos, però no entre sons i cares d'altres animals, com per exemple ànecs (Vouloumanos, Druhen, Hauser i Huizink 2009).

Aquest recull d'estudis mostra que des de la infantesa les pistes audiovisuals són percebudes i utilitzades en la percepció dels estímuls lingüístics. Als apartats següents es presenten estudis que exploren una nova manera d'enfocar la percepció audiovisual de la parla: els patrons atencionals a la cara d'un parlant.

\footnotetext{
1 En presentar visualment la síl-laba /ga/ i auditivament la síl·laba /ba/ els adults perceben la síl·laba /da/, que correspon a la combinació de la informació auditiva i visual presentada (McGurk i MacDonald 1976).
} 


\section{Els PATRONS D’ATENCIÓ-MIRADA A LA CARA D’UN PARLANT}

Des dels estudis clàssics de Yarbus (1967), sabem que els adults mirem majoritàriament als ulls de la persona que parla, amb l'objectiu d'extreure'n al màxim d'informació social i expressivitat (Birmingham i Kingstone 2009). És només quan les pistes visuals de la parla es tornen rellevants que canviem la nostra atenció i ens focalitzem directament a la boca del parlant. Estudis previs demostren que aquest canvi d'atenció i focalització a la boca es produeix quan el so està emmascarat amb soroll (Lansing i McConkie 2003; Vatikiotis-Bateson, Eigsti, Yanoyi i Munhall 1998), en els moments en què la parla esdevé més rellevant (Võ, Smith, Mital i Henderson 2012) o bé quan els participants han de resoldre una tasca específica que involucra per exemple una atenció especial al contingut del discurs (Barenholtz, Mavica i Lewkowicz 2016; Buchan, Paré i Munhall 2007; Lusk i Mitchel 2016). Pel que fa a l'adquisició del llenguatge, altres estudis recents han analitzat la manera com els infants exploren la cara d'un parlant i els indicis que ens proporcionen sobre el moment o fase en què es troben, i també sobre possibles problemes d'aprenentatge relacionats amb la percepció audiovisual de la parla.

\subsection{Desenvolupament del patró d'atenció selectiva a una cara parlant}

En un estudi pioner, Lewkowicz i Hansen-Tift (2012) van estudiar com els nadons exploren la cara d'una persona parlant. Utilitzant la tècnica de l'oculometria (eye tracker) van observar que els infants de 4 mesos focalitzen l'atenció als ulls de la persona. Entre els 4 i els 8 mesos canvien l'atenció i se centren més en la boca de la parlant. Els autors van relacionar aquest canvi de focalització amb l'aparició dels primers balbotejos, i d'aquesta manera van interpretar que els infants atenien les pistes audiovisuals redundants de la parla -és a dir, la boca- amb l'objectiu de millorar l'adquisició dels sons de la llengua materna. Finalment, els infants de 12 mesos perdien la tendència d'observar la boca i exploraven a parts iguals la boca i els ulls de la parlant, la qual cosa els permetia accedir també als senyals socials que són fonamentals en el desenvolupament cognitiu i comunicatiu (Pons, Bosch i Lewkowicz 2019). Així doncs, aquest va ser el primer estudi en relacionar els patrons d'exploració facial durant el primer any de vida amb el desenvolupament lingüístic dels infants.

Més tard, durant el segon any de vida, un estudi de Hillairet de Boisferon i col-laboradors (2018) demostra que els infants segueixen atenent preferentment la boca, $\mathrm{i}$ ho relacionen amb el fet que els infants de 14 a 18 mesos estan en la fase d'explosió de vocabulari, cosa que torna a fer rellevants les pistes audiovisuals de la boca. En la mateixa línia, un altre estudi indica que, efectivament, el pic de fixacions a la boca és al voltant dels 18 mesos, quan comença un lent decrement de la boca a favor dels ulls (Jones i Klin 2013).

Curiosament, no és fins que la llengua -o llengües- es pot considerar pràcticament assolida, cap als 6 anys d'edat, que desapareix la preferència per la boca i l'atenció dels nens queda distribuïda entre els ulls i la boca de la parlant (Birulés, Bosch, Brieke, Pons i Lewkowicz 2018; Król 2018; Morin-Lessard, Poulin-Dubois, Segalowitz i Heinlein 2019; Nakano et al. 2010). Tot i així, comparat amb la clara preferència pels ulls que mostren els adults, el fet que els nens encara explorin equitativament els ulls i la boca suggereix que la percepció audiovisual del llenguatge no està encara del tot desenvolupada i que són en un punt mig entre els infants i els adults.

Per últim, en l'edat adulta els patrons de mirada poden ser un mirall de l'estat cognitiu dels participants. Per exemple, en un estudi de Thompson \& Malloy (2004) es reporta 
una tornada de l'atenció a la boca en participants d'edat avançada (mitjana de 70 anys) en comparació amb participants més joves (mitjana de 23 anys), que exhibeixen una forta preferència pels ulls. En la mateixa línia i similar als estudis anteriorment reportats sobre el processament de la parla amb soroll, un estudi recent demostra que els adults també incrementen l'atenció a la boca quan perceben una llengua diferent de la materna en l'interlocutor, tot i ser-ne perfectament competents (Birulés, Bosch, Pons i Lewkowicz, in prep). Aquests estudis reforcen la idea que els adults també utilitzen l'estratègia d'atendre més la boca quan la comprensió del missatge resulta dificultosa i se'ls requereix un esforç més gran de processament.

Així doncs, en combinació, aquests estudis relacionen - tot i que indirectament- els patrons d'exploració facial amb el desenvolupament lingüístic i l'esforç de processament, des de la infantesa fins a l'edat adulta. Si aquesta relació és correcta, seria possible que la modulació d'un factor lingüístic impliqués també un patró d'exploració diferent de la cara d'un parlant?

\subsection{La influència d'un entorn bilingüe en l'atenció selectiva a la cara del parlant}

Subseqüentment a l'estudi de Lewkowicz i Hansen-Tift (2012) -en què es va definir per primera vegada el patró de desenvolupament d'exploració facial durant el primer any de vida-, Pons, Bosch, i Lewkowicz (2015) van comparar els patrons d'exploració facial d'infants provinents d'entorns monolingües amb els patrons d'infants d'entorns bilingües. El factor del bilingüisme no és tan sols una realitat lingüística diferent sinó que pot plantejar una tasca complexa per als infants: per aprendre dues llengües a la vegada cal primer aprendre a diferenciar-les, i així construir els dos sistemes lingüístics per separat. Considerant els estudis esmentats, seria esperable que els infants d'entorns bilingües explotessin en major mesura totes aquelles pistes que poguessin ajudar-los, com per exemple les pistes redundants i audiovisuals de la boca. Per respondre a aquesta qüestió, Pons, Bosch i Lewkowicz (2015) van estudiar les diferències en el patró de mirada a la cara d'un parlant entre infants monolingües i infants bilingües català-castellà, als 4, 8 i 12 mesos, i van mostrar que efectivament existien diferències rellevants entre les dues poblacions. Per començar, mentre que els infants monolingües de 4 mesos atenien més els ulls, els infants bilingües atenien per igual ulls i boca. Als 8 mesos d'edat, els dos grups passaven la major part del temps mirant la boca de la parlant. Finalment, als 12 mesos, els infants bilingües mantenien la preferència per la boca de la parlant, mentre que els monolingües mostraven un patró equilibrat d'atenció entre els ulls i la boca. Aquests resultats van demostrar que els infants bilingües prestaven atenció a les pistes redundants i audiovisuals de la boca des d'una edat més primerenca i durant més temps que els infants monolingües, la qual cosa fa pensar que, efectivament, els bilingues utilitzen les pistes audiovisuals per ajudar-los a percebre i processar un senyal lingüístic més complex.

Posteriorment, diversos estudis han ampliat aquest descobriment i han permès entendre aquest fenomen amb més detall. Per exemple, Ayneto i Sebastián-Gallés (2016) van observar que, als 8 mesos, els infants bilingües passaven més temps que els monolingües observant la boca d'una cara, encara que no estigués parlant sinó fent diferents expressions afectives, la qual cosa indicava que l'accentuada preferència per la boca dels infants bilingües es generalitzava també a altres tipus de situacions no lingüístiques. En la mateixa línia, Fort i col-laboradors (2017) van demostrar que els infants bilingües de 15 mesos no són capaços d'anticipar un moviment ocular de la cara d'un parlant, mentre que els monolingües sí que en són capaços. Les autores van 
interpretar que aquesta diferència era conseqüència de la forta atracció per la boca dels bilingües, que els fa desestimar o no posar tanta atenció a les altres zones de la cara. En combinació, aquests estudis corroboren que els infants provinents d'entorns bilingües presten més atenció a la boca d'un parlant, i deixen palès que un factor solament lingüístic pot modular també l'exploració facial i la utilització de les pistes audiovisuals.

Per acabar, cal ressaltar que les mostres bilingües dels estudis esmentats són d'entorn català-castellà, dues llengües properes (rítmicament i fonològicament) i més difícils de diferenciar pels infants que les llengües distants. Estudis fets amb infants bilingües de llengües distants (anglès-francès o anglès-altres) no han trobat diferències clares entre els patrons d'exploració facial dels bilingües i dels monolingües (Morin-Lessard et al. 2019 i Tsang, Atagi i Johnson 2018). Per aquest motiu, un estudi recent de Birulés i col-laboradors (2018) ha explorat específicament si la tipologia de bilingüisme pot tenir un efecte sobre els patrons d'atenció a una cara parlant. En aquest estudi es van comparar dues poblacions bilingües: l'una amb dues llengües rítmicament i fonològicament properes (català i castellà) i l'altra amb dues llengües llunyanes (castellà i anglès, alemany, suec o àrab, entre d'altres) en dos grups d'edat, infants de 15 mesos i nens de 5 anys. Els resultats indiquen que en ambdós grups d'edat, els bilingües de llengües properes mostren més atenció a la boca de la parlant que els bilingües de llengües llunyanes. A més a més, el fet que les diferències entre grups persisteixin encara en nens de 5 anys suggereix que els factors lingüístics afecten el desenvolupament dels estratagemes d'exploració facial d'una manera general.

El fet que la modulació d'un factor lingüístic com és el bilingüisme provoqui també diferències en l'atenció selectiva a la cara d'una parlant suggereix que ambdós factors estan fortament relacionats, i dona suport a les interpretacions més lingüístiques dels estudis de patrons d'atenció a una cara -com per exemple, que els infants de 4 a 8 mesos canvien la seva atenció dels ulls a la boca perquè tenen un interès creixent a aprendre el llenguatge, i així s'ajuden de les pistes audiovisuals de la boca (Lewkowicz i Hansen-Tift 2012). Així ho demostren també diversos estudis que han correlacionat el temps d'atenció a la boca durant el primer any de vida amb el posterior desenvolupament del llenguatge (Tenenbaum et al. 2015, Tsang et al. 2018 i Young et al. 2009).

\section{CONCLUSIONS}

Així, el comú denominador que s'extrau d'aquests estudis és que des del primer any de vida i fins a l'edat adulta la informació audiovisual del llenguatge és crucial per a l'aprenentatge. Aquest fet queda específicament demostrat a través dels patrons de mirada a la cara d'una persona que ens parla, que aporten una informació molt rellevant de quines són les prioritats atencionals de cada situació, que depenen de factors interns com l'edat, les capacitats cognitives o la llengua materna / llengües maternes, i també de factors externs com la tasca concreta a solucionar, la llengua produïda o la qualitat de la informació d'arribada. A la vegada, entendre les prioritats atencionals durant el desenvolupament també ens informa sobre quines pistes audiovisuals de la cara s'aprofiten més en cada moment i per quins motius.

Per acabar, un aspecte crucial d'aquests estudis és que se'n poden derivar implicacions clíniques rellevants. Estudis del desenvolupament de l'atenció a la cara d'un parlant han demostrat que les variacions importants dels patrons anteriorment descrits (i. $e$. desenvolupament normotípic) poden estar també relacionats amb diversos trastorns, com per exemple el trastorn de l'espectre autista (Chawarska, MacAri i Shic 2012; Jones i Klin 2013; Nakano et al. 2010) o el trastorn específic del llenguatge (Pons, Sanz-Torrent, Ferinu, Birulés i Andreu 2018). Aquests estudis suggereixen que es podrien utilitzar 
mesures d'atenció selectiva per detectar o diagnosticar trastorns del desenvolupament, i també subratllen la importància d'incloure materials audiovisuals en les teràpies d’aprenentatge lingüístic (Heikkilä et al. 2018, Irwin i DiBlasi 2017).

\section{AgraÏMENTS}

Agraïments a Teresa Fernàndez per la correcció del text.

\section{REFERÈNCIES BIBLIOGRÀFIQUES}

Ayneto, Alba; Sebastián-Gallés, Nuria (2016). "The influence of bilingualism on the preference for the mouth region of dynamic faces». Developmental Science, 1-11. $<$ https://doi.org/10.1111/desc.12446> [Consulta: 27 maig 2019].

BAHRICK, LORRAINE E.; LICKLITER, ROBERT (2012). «The role of intersensory redundancy in early perceptual, cognitive, and social development». BREMnER, A. J.; LEWKOWICZ, D. J.; SPENCE, C. (eds.). Multisensory Development (183-206). New York, NY, US: Oxford University Press. <https://doi.org/10.1093/acprof:oso/9780199586059.003.0008> [Consulta: 27 maig 2019].

Barenholtz, Elan; Mavica, LaUREN; LeWKowicz, DaVID J. (2016). «Language familiarity modulates relative attention to the eyes and mouth of a talker». Cognition, 147, 100-105. $<$ https://doi.org/10.1016/j.cognition.2015.11.013> [Consulta: 27 maig 2019].

Birmingham, Elina; Kingstone, Alan (2009). «Human social attention: A new look at past, present, and future investigations». Annals of the New York Academy of Sciences, 1156, 118-140. <https://doi.org/10.1111/j.1749-6632.2009.04468.x > [Consulta: 27 maig $2019]$.

Birulés, Joan; Bosch, LaUra; Brieke, Ricarda; Pons, Ferran; Lewkowicz, David J. (2018). "Inside Bilingualism: Language Background Modulates Selective Attention to a Talker's Mouth». Developmental Science, e12755. <https://doi.org/10.1111/desc.12755> [Consulta: 27 maig 2019].

Birulés, Joan; Bosch, Laura; Pons, Ferran; Lewkowicz, David J. (in prep.). «Even the highly proficient non-native speakers rely on the mouth when processing non-native speech».

Bremner, ANdrew J.; Lewkowicz, David J.; Spence, Charles (2012). «Multisensory Development». Oxford University Press. $<$ https://doi.org/10.1093/acprof:0so/9780199586059.001.0001> [Consulta: 27 maig 2019].

Buchan, Julié; Paré, Martin; Munhall, Kevin G. (2007). «Spatial statistics of gaze fixations during dynamic face processing». Social Neuroscience, 2(1), 1-13. <https://doi.org/10.1080/17470910601043644> [Consulta: 27 maig 2019].

Chandrasekaran, Chandramouli; Trubanova, Andrea; Stillittano, Sébastian; CAPLIER, AlicE; GHAZANFAR, ASIF A. (2009). "The natural statistics of audiovisual speech». PLoS Computational Biology, 5(7). $<$ https://doi.org/10.1371/journal.pcbi.1000436> [Consulta: 27 maig 2019].

Chazanfar, Katarzyna; Macari, Suzanne; Shic, Frederick (2012). «Context modulates attention to social scenes in toddlers with autism». Journal of Child Psychology and Psychiatry and Allied Disciplines, 53(8), 903-913. <https://doi.org/10.1111/j.14697610.2012.02538. $x>$ [Consulta: 27 maig 2019].

CotTon, JAск C. (1935). "Normal "Visual Hearing"». Science, 82(2138), 592-593. $<$ https://science-sciencemag-org.sire.ub.edu/content/82/2138/592> [Consulta: 27 maig 2019]. 
DODD, BARBARA (1979). «Lip reading in infants: Attention to speech presented in- and out-of-synchrony». Cognitive Psychology, 11(4), 478-484. <https://doi.org/10.1016/0010-0285(79)90021-5> [Consulta: 27 maig 2019].

Fort, Mathilde; Ayneto-Gimeno, Alba; Escrichs, Anira; Sebastián-Gallés, Nuria (2017). «Impact of Bilingualism on Infants' Ability to Learn From Talking and Nontalking Faces». Language Learning, 1-27. < https://doi.org/10.1111/lang.12273> [Consulta: 27 maig 2019].

Heikkilä, Jenni; lonka, Eila; Meronen, Auli; Tuovinen, Sisko; Eronen, RaiJa; Leppänen, PaAvo H.; Richardson, Ulla; Ahonen, Timo; Tirppana, Kaisa (2018). «The effect of audiovisual speech training on the phonological skills of children with specific language impairment (SLI)». Child Language Teaching and Therapy. $<$ https://doi.org/10.1177/0265659018793697> [Consulta: 29 maig 2019].

Hillairet De Boisferon, Anne; Tift, Amy H.; Minar, Nicholas J.; Lewkowicz, David J. (2018). «The redeployment of attention to the mouth of a talking face during the second year of life». Journal of Experimental Child Psychology, 172, 189-200. $<$ https://doi.org/10.1016/j.jecp.2018.03.009> [Consulta: 29 maig 2019].

IRWIN, JULIA; DiBLASI, LORI (2017). «Audiovisual speech perception: A new approach and implications for clinical populations». Linguistics and Language Compass, 11(3), 77-91. $<$ https://doi.org/10.1111/lnc3.12237> [Consulta: 29 maig 2019].

JONES, WARREN; Klin, Ami (2013). "Attention to eyes is present but in decline in 2-6month-old infants later diagnosed with autism». Nature, 504(7480), 427-431. $<$ https://doi.org/10.1038/nature12715> [Consulta: 29 maig 2019].

KRÓL, MAGdalena E. (2018). "Auditory noise increases the allocation of attention to the mouth, and the eyes pay the price: An eye-tracking study». PLOS ONE, 13(3), 1-14. $<$ https://doi.org/10.1371/journal.pone.0194491> [Consulta: 29 maig 2019].

Kuhl, P. K.; Meltzoff, A. N. (1982). «The bimodal perception of speech in infancy». Science. $<$ https://doi.org/10.1126/science.7146899> [Consulta: 29 maig 2019].

LANSIng, Charissa R.; Mcconkie, George W. (2003). «Word identification and eye fixation locations in visual and visual-plus-auditory presentations of spoken sentences». Perception \& Psychophysics, 65(4), 536-552. <https://doi.org/10.3758/BF03194581> [Consulta: 29 maig 2019].

LewKowicz, David J.; HANSEN-Tift, Amy M. (2012). «Infants deploy selective attention to the mouth of a talking face when learning speech». Proceedings of the National Academy of Sciences of the United States of America, 109(5), 1431-1436. <https://doi.org/10.1073/pnas.1114783109> [Consulta: 29 maig 2019].

Lusk, Laina G.; Mitchel, AAron D. (2016). «Differential Gaze Patterns on Eyes and Mouth During Audiovisual Speech Segmentation». Frontiers in Psychology, 7, 52. $<$ https://doi.org/10.3389/fpsyg.2016.00052> [Consulta: 29 maig 2019].

MCGURK, HARRY; MACDONALD, JoHN (1976). «Hearing lips and seeing voices». Nature, 264(5588), 691-811. <https://doi.org/10.1038/264746ao> [Consulta: 29 maig 2019].

Meredith, M. A.; STEIN, B. E. (1986). «Visual, auditory, and somatosensory convergence on cells in superior colliculus results in multisensory integration». Journal of Neurophysiology, 56(3), 640-662. <https://doi.org/10.1152/jn.1986.56.3.640 > [Consulta: 29 maig 2019].

Morin-Lessard, Elisabeth; Poulin-Dubois, Diane; Segalowitz, Norman; ByersHEINLEIN, KRISTA (2019). "Selective attention to the mouth of talking faces in monolinguals and bilinguals aged 5 months to 5 years.». Developmental Psychology, 16o. <http://www.doi.org/10.17605/OSF.IO/IKVYR> [Consulta: 29 maig 2019]. 
Nakano, Tamami; Tanaka, Kyoko; Endo, Yuuki; Yamane, Yui; Yamamoto, Takahiro; Nakano, Yoshiaki; Ohta, Haruhisa; Kato, Nobumasa; KitaZawa, Shigeru (2010). «Atypical gaze patterns in children and adults with autism spectrum disorders dissociated from developmental changes in gaze behaviour». Proceedings of the Royal Society B: Biological Sciences, 277(1696), 2935-2943. <https://doi.org/10.1098/rspb.2010.0587> [Consulta: 29 maig 2019].

Patterson, Michelle L.; Werker, Janet F. (2003). «Two-month-old infants match phonetic information in lips and voice». Developmental Science, 6(2), 191-196. $<$ https://doi.org/10.1111/1467-7687.00271> [Consulta: 29 maig 2019].

Pons, FERran; Bosch, LAURA; LEWKowicz, DAVID J. (2015). «Bilingualism Modulates Infants' Selective Attention to the Mouth of a Talking Face». Psychological Science, 26(4), 490-498. <https://doi.org/10.1177/0956797614568320> [Consulta: 29 maig 2019].

Pons, Ferran; Bosch, LaUra; Lewkowicz, David J. (2019). «Twelve-month-old infants' attention to the eyes of a talking face is associated with communication and social skills». Infant Behavior and 80velopment, 54, 84. $<$ https://doi.org/10.1016/j.infbeh.2018.12.003> [Consulta: 29 maig 2019].

Pons, Ferran; LewKowicz, David J.; Soto-Faraco, SAlvador; SEbastián-GallÉs, NuRia (2009). "Narrowing of intersensory speech perception in infancy». Proceedings of the National Academy of Sciences of the United States of America, 106(26), 10598-10602. $<$ https://doi.org/10.1073/pnas.0904134106 > [Consulta: 29 maig 2019].

Pons, Ferran; SAnz-Torrent, Monica; Ferinu, Laura; Birulés, Joan; Andreu, LLORENC (2018). "Children With SLI Can Exhibit Reduced Attention to a Talker's Mouth». Language Learning, (68), 180-192. <https://doi.org/10.1111/lang.12276> [Consulta: 29 maig 2019].

Poulin-Dubois, Diane; Serbin, lisa A.; Kenyon, Brenda; Derbyshire, Alison (1994). «Infants' Intermodal Knowledge About Gender». Developmental Psychology, 30(3), 436-442. <https://doi.org/10.1037/0012-1649.30.3.436> [Consulta: 29 maig 2019].

RisberG, A.; LubKer, J. (1978). «Prosody and speech-reading». Quarterly Progress and Status Report, $\quad 4, \quad 1-16$. <http://www.speech.kth.se/prod/publications/files/qpsr/1978/1978_19_4_oo1o16.pdf $>$ [Consulta: 29 maig 2019].

Rosenblum, Lawrence D.; Schmuckler, Mark A.; Johnson, Jennifer A. (1997). «The McGurk effect in infants». Perception \& Psychophysics, 59(3), 347-357. $<$ https://doi.org/10.3758/BF03211902> [Consulta: 29 maig 2019].

SANDERS, DereK A.; GoOdrich, Sharon. J. (1971). «The Relative Contribution of Visual and Auditory Components of Speech to Speech Intelligibility under Varying Conditions of Frequency Distortion». Journal of Speech Language and Hearing Research, 14(1), 154159. <https://doi.org/10.1121/1.2143572> [Consulta: 29 maig 2019].

SumbY, W. H.; PolLACK, IRWIN (1954). «Visual Contribution to Speech Intelligibility in Noise». The Journal of the Acoustical Society of America, 26(2), 212-215. $<$ https://doi.org/10.1121/1.1907309> [Consulta: 29 maig 2019].

Tenenbaum, Elena J.; Sobel, David M.; Sheinkopf, Stephen J.; Malle, Bertram F.; MORGAN, JAMES L. (2015). "Attention to the mouth and gaze following in infancy predict language development». Journal of Child Language, 42(6), 1173-1190. <https://doi.org/10.1017/S0305000914000725> [Consulta: 29 maig 2019].

Thompson, L. A.; Malloy, D. (2004). «Attention resources and visible speech encoding in older and younger adults». Experimental Aging Research, 30(3), 241-252. <https://doi.org/10.1080/03610730490447877> [Consulta: 29 maig 2019]. 
Tsang, Tawny; Atagi, Natsuki; Johnson, ScotT P. (2018). «Selective attention to the mouth is associated with expressive language skills in monolingual and bilingual infants». Journal of Experimental Child Psychology, 169, 93-109. $<$ https://doi.org/10.1016/j.jecp.2018.01.002> [Consulta: 29 maig 2019].

Vatikiotis-Bateson, Eric; Eigsti, Inge-Marie; Yano, Sumio; Munhall, Kevin G. (1998). «Eye movement of perceivers during audiovisual speech perception». Perception \& Psychophysics, 6o(6), 926-940. <https://doi.org/10.3758/BFo3211929> [Consulta: 29 maig 2019].

Võ, Melissa L.-H.; Smith, Tim J.; Mital, Parag K.; Henderson, John M. (2012). «Do the eyes really have it? Dynamic allocation of attention when viewing moving faces». Journal of Vision, 12(13), 3-3. < https://doi.org/10.1167/12.13.3> [Consulta: 29 maig 2019].

Vouloumanos, Athena; Druhen, Madelynn J.; Hauser, Marc D.; Huizink, Anouk T. (2009). "Five-month-old infants' identification of the sources of vocalizations». Proceedings of the National Academy of Sciences of the United States of America, 106(44), 18867-18872. <https://doi.org/10.1073/pnas.0906049106> [Consulta: 29 maig 2019].

WALKER-ANDREWS, ARLENE S. (1997). «Infants' perception of expressive behaviors: Differentiation of multimodal information». Psychological Bulletin, 121(3), 437-456. $<$ https://doi.org/10.1037/0033-2909.121.3.437> [Consulta: 29 maig 2019].

Weikum, Whitney M.; Vouloumanos, Athena; Navarra, Jordi; Soto-Faraco, SAlVAdOR; SEbastián-GallÉS, Núria; Werker, JANET F. (2007). "Visual language discrimination in infancy». Science (New York, N.Y.), 316(5828), 1159. $<$ https://doi.org/10.1126/science.1137686> [Consulta: 29 maig 2019].

YARBUS, A. L. (1967). «Eye movements and vision». New York, New York, USA: Plenum Press. <https://doi.org/10.1016/0028-3932(68)90012-2> [Consulta: 29 maig 2019].

Yehia, Hani; Rubin, Philip; Vatikiotis-Bateson, ERIC (1998). «Quantitative association of vocal-tract and facial behavior». Speech Communication, 26(1-2), 23-43. $<$ https://doi.org/10.1016/S0167-6393(98)oo048-X > [Consulta: 29 maig 2019].

Young, Gregory S.; Merin, Noah; Rogers, Sally J.; Ozonoff, SAlly (2009). «Gaze behavior and affect at 6 months: Predicting clinical outcomes and language development in typically developing infants and infants at risk for autism». Developmental Science, 12(5), 798-814. <https://doi.org/10.1111/j.1467-7687.2009.00833.x > [Consulta: 29 maig 2019]. 